\title{
On a Relation Between the Zagreb Indices
}

\author{
Sanja Stevanović \\ University of Niš, Faculty of Civil Engineering and Architecture, \\ Aleksandra Medvedeva bb, 18000 Niš, Serbia \\ (E-mail: sanja_stevanovic@yahoo.com)
}

RECEIVED APRIL 8, 2009; REVISED JULY 24, 2009; ACCEPTED JULY 28, 2009

\begin{abstract}
Let $G=(V, E)$ be a simple graph with $n=|V|$ vertices and $m=|E|$ edges. The first and the second Zagreb index are defined as $M_{1}=\sum_{u \in V} d_{u}^{2}$ and $M_{2}=\sum_{u v \in E} d_{u} d_{v}$, where $d_{u}$ is the degree of vertex $u$. Professor Pierre Hansen at the International Academy of Mathematical Chemistry Meeting in 2006 conjectured that $\frac{M_{1}}{m} \leq \frac{M_{2}}{n}$ holds for all simple graphs. While the conjecture is true for trees, unicyclic and chemical graphs, several counterexamples appeared in the literature. Here we extend the construction of counterexamples by showing that we may add a sufficiently large star to any graph $G$ with $m \geq n+\delta$ to obtain a counterexample.

For the variable Zagreb indices ${ }^{\lambda} M_{1}=\sum_{u \in V} d_{u}^{2 \lambda}$ and ${ }^{\lambda} M_{2}=\sum_{u v \in E} d_{u}^{\lambda} d_{v}^{\lambda}$, we prove that any graph $G$ can be extended by a suitably large star so that ${ }^{\lambda} M_{1} / n>{ }^{\lambda} M_{2} / m$ when $0<\lambda<1$, and ${ }^{\lambda} M_{1} / n<{ }^{\lambda} M_{2} / m$ when $\lambda<0$ or $\lambda>1$. (doi: $10.5562 / \mathrm{cca} 1579$ )
\end{abstract}

Keywords: Molecular structure descriptor, Zagreb indices.

\section{INTRODUCTION}

Let $G=(V, E)$ be a simple graph with $n=|V|$ vertices and $m=|E|$ edges. For $u \in V$, let $N(u)$ denote the set of neighbors of $u, d_{\mathrm{u}}=|N(u)|$ be the degree of $u$, and let $\Delta=\max _{u \in V} d_{u}$ and $\delta=\min _{u \in V} d_{u}$ be the maximum and the minimum vertex degree, respectively. The first $\mathrm{Za}$ greb index $M_{1}$ and the second Zagreb index $M_{2}$ are defined as

$$
M_{1}=\sum_{u \in V} d_{u}^{2} \text { and } M_{2}=\sum_{(u, v) \in E} d_{u} d_{v} .
$$

The Zagreb indices appeared as two terms in the topological formula for the total $\pi$-electron energy of conjugated molecules in $1972 .{ }^{1}$ However, the Zagreb indices as such were first reported in $1975 .^{2}$ The surveys of the properties of $M_{1}$ and $M_{2}$ are given in Refs. 3 and 4. With the help of the computer system AUTOGRAPHIX, ${ }^{5}$ the following conjecture comparing the Zagreb indices was proposed by Professor Pierre Hansen at the International Academy of Mathematical Chemistry Meeting in 2006: Conjecture 1. For all simple connected graphs G,

$$
\frac{M_{1}}{m} \leq \frac{M_{2}}{n}
$$

and the bound is tight for complete graphs.
This conjecture was proved for chemical graphs, ${ }^{6}$ for trees, ${ }^{7}$ for unicyclic graphs, ${ }^{8}$ for subdivided graphs, ${ }^{9}$ and for graphs with $\Delta-\delta \leq 3, \delta \neq 2$, in Ref. 10 , by generalizing the method from Ref. 6 .

For $u \in V$, let $D_{u}=\sum_{v \in N(u)} d_{v}$ be the 2-degree of vertex $u$. Then

$$
M_{1}=\sum_{v \in V} d_{v}^{2}=\sum_{v \in V} d_{v}|N(v)|=\sum_{u \in V} \sum_{v \in N(u)} d_{v}=\sum_{u \in V} D_{u},
$$

so that

$$
\frac{M_{1}}{n}=\sum_{u \in V} \frac{1}{n} D_{u} .
$$

On the other hand,

$$
2 M_{2}=\sum_{u v \in E} 2 d_{u} d_{v}=\sum_{u \in v} d_{u} \sum_{v \in N(u)} d_{v}=\sum_{u \in V} d_{u} D_{u},
$$

so that

$$
\frac{M_{2}}{m}=\frac{2 M_{2}}{2 m}=\sum_{u \in V} \frac{d_{u}}{2 m} D_{u} .
$$

Since $\sum_{u \in V} \frac{1}{n}=\sum_{u \in V} \frac{d_{u}}{2 m}=1$, both expressions $\frac{M_{1}}{n}$ and $\frac{M_{2}}{m}$ represent convex linear combinations of the same set of values $\left\{D_{u}: u \in V\right\}$, which may serve to explain a close similarity between them. 
However, Conjecture 1 is not true in general. Let $K_{n}$ denote a complete graph on $n$ vertices, $K_{m, n}$ a complete bipartite graph with $m+n$ vertices, and $S_{n} \cong K_{1, n-1}$ a star on $n$ vertices. Several counterexamples appeared earlier: the smallest counterexample $S_{6} \cup K_{3}$ was found in Ref. 6, together with a connected counterexample on 46 vertices and 110 edges; next, a connected bicyclic counterexample with 19 vertices was found in Ref. 9, and $K_{2,5} \cup K_{4}$ is a counterexample found in Ref. 10 with $\Delta=5, \delta=2$.

Thus, while Conjecture 1 holds for several graph classes, it also has a number of counterexamples. Our goal here is to show that Conjecture 1 may hold only for very restricted graph classes, as suggested in the next section where we show that a sufficiently large star can be added to almost any graph in order to obtain a counterexample to Conjecture 1 . We then extend this reasoning to variable Zagreb indices in Section 3.

\section{ALMOST ANY GRAPH IS CONTAINED WITHIN A COUNTEREXAMPLE}

For a graph $G$, its vertex $u$, and an integer $a \geq 2$, let $G_{u, a}$ be the graph obtained from $G$ and $S_{a+1}$ by identifying vertex $u$ with one of the leaves of $S_{a+1}$. We prove the following

Theorem 2. Let $G$ be a simple graph such that $m \geq n+\delta$. Then there exist a vertex $u$ of $G$ and a real number $A$, such that for any $a>A$, the graph $G_{u, a}$ is a counterexample to Conjecture 1 .

Proof: We have that the number of vertices and edges of $G_{u, a}$ are equal to $n\left(G_{u, a}\right)=n+a$ and $m\left(G_{u, a}\right)=m+a$, respectively. Further,

$$
\begin{aligned}
M_{1}\left(G_{u, a}\right) & =M_{1}(G)+a^{2}+(a-1) 1^{2}+(d u+1)^{2}-d_{u}^{2} \\
& =M_{1}(G)+a^{2}+a+2 d_{u} .
\end{aligned}
$$

Next,

$$
\begin{gathered}
M_{2}\left(G_{u, a}\right)=M_{2}(G)+(a-1) \cdot a \cdot 1+a\left(d_{u}+1\right)+ \\
\sum_{v \in N_{u}}\left(d_{u}+1\right) d_{v}-\sum_{v \in N_{u}} d_{u} d_{v}=M_{2}(G)+a^{2}+a d_{u}+D_{u} .
\end{gathered}
$$

The inequality

$$
\frac{M_{2}\left(G_{u, a}\right)}{m\left(G_{u, a}\right)}<\frac{M_{1}\left(G_{u, a}\right)}{n\left(G_{u, a}\right)}
$$

is equivalent to

$$
\begin{aligned}
& \left(M_{2}(G)+a^{2}+a d_{u}+D_{u}\right)(n+a)<\left(M_{1}(G)+\right. \\
& \left.a^{2}+a+2 d_{u}\right)(m+a)
\end{aligned}
$$

i.e., to

$$
\begin{aligned}
& 0<f(a)=a^{2}\left(m+1-n-d_{u}\right)+ \\
& a\left(M_{1}(G)+m-M_{2}(G)-D_{u}-d_{u}(n-2)\right)+ \\
& \left(M_{1}(G) m+2 d_{u} m-M_{2}(G) n-D_{u} n\right) .
\end{aligned}
$$

Thus, $f(a)$ is a quadratic function in $a$ with constant coefficients for any fixed choice of $u$. Now, choose $u$ to be a vertex of minimum degree in $G$, i.e., $d_{u}=\delta$. From $m \geq n+\delta$ we have that the coefficient of $a^{2}$ in $f(a)$ is strictly positive. Therefore,

$$
\lim _{a \rightarrow+\infty} f(a)=+\infty
$$

so there exists a real number $A$ such that $f(a)>0$ for any $a>A$. Graph $G_{u, a}$ is then a counterexample to Conjecture 1 for any $a>A$.

Thus, we may add a sufficiently large star to any graph $G$ with $m \geq n+\delta$ to obtain a counterexample to Conjecture 1 . For example, if $n \geq 6$ and $\delta \geq 3$, we always have that $m \geq n \delta / 2 \geq n+\delta$, so Theorem 2 can be applied to any such graph.

\section{A RELATION BETWEEN THE VARIABLE ZAGREB INDICES}

The Zagreb indices have been generalized to the variable Zagreb indices ${ }^{11}$

$$
{ }^{\lambda} M_{1}=\sum_{u \in V} d_{u}^{2 \lambda} \text { and }{ }^{\lambda} M_{2}=\sum_{u v \in E} d_{u}^{\lambda} d_{v}^{\lambda}
$$

The properties of the variable Zagreb indices were discussed in Refs. 12 and 13. For $\lambda=0$ we have ${ }^{0} M_{1}=n$, ${ }^{0} M_{2}=m$, while for $\lambda=1$ we obtain the standard Zagreb indices. Theorem 2 above may be extended to the variable Zagreb indices in the following way.

Theorem 3. Let $G$ be a simple graph. If $0<\lambda<1$, then for each vertex $u$ of $G$ there exists a real number $A_{u}$, such that for any $a>A_{u}$, the graph $G_{u, a}$ satisfies

$$
\frac{{ }^{\lambda} M_{1}\left(G_{u, a}\right)}{n\left(G_{u, a}\right)}>\frac{{ }^{\lambda} M_{2}\left(G_{u, a}\right)}{m\left(G_{u, a}\right)} .
$$

If $\lambda<0$ or $1<\lambda$ then for each vertex $u$ of $G$ there exists a real number $A_{u}$, such that for any $a>A_{u}$, the graph $G_{u, a}$ satisfies

$$
\frac{{ }^{\lambda} M_{1}\left(G_{u, a}\right)}{n\left(G_{u, a}\right)}<\frac{{ }^{\lambda} M_{2}\left(G_{u, a}\right)}{m\left(G_{u, a}\right)} .
$$

Proof: Let $u$ be an arbitrary vertex of $G$. Then 


$$
\begin{aligned}
{ }^{\lambda} M_{1}\left(G_{u, a}\right) & ={ }^{\lambda} M_{1}(G)+a^{2 \lambda}+(a-1) \cdot 1^{2 \lambda}+\left(d_{u}+1\right)^{2 \lambda}-d_{u}^{2 \lambda} \\
& =a^{2 \lambda}+a+c_{0},
\end{aligned}
$$

for a constant

$$
c_{0}={ }^{\lambda} M_{1}(G)-1+\left(d_{u}+1\right)^{2 \lambda}-d_{u}^{2 \lambda} .
$$

Next,

$$
\begin{gathered}
{ }^{\lambda} M_{2}\left(G_{u, a}\right)={ }^{\lambda} M_{2}(G)+(a-1) \cdot a^{\lambda} \cdot 1^{\lambda}+a^{\lambda}\left(d_{u}+1\right)^{\lambda}+ \\
\sum_{v \in N_{u}}\left(\left(d_{u}+1\right)^{\lambda}-d_{u}^{\lambda}\right) d_{v}^{\lambda}=a^{\lambda+1}+a^{\lambda} c_{1}+c_{2},
\end{gathered}
$$

for constants

$$
\begin{aligned}
& c_{1}=\left(d_{u}+1\right)^{\lambda}-1 \text { and } \\
& c_{2}={ }^{\lambda} M_{2}(G)+\sum_{v 0 N_{u}}\left(d_{u}+1\right)^{\lambda}
\end{aligned}
$$

The sign of

$$
\frac{{ }^{\lambda} M_{2}\left(G_{u, a}\right)}{m\left(G_{u, a}\right)}-\frac{{ }^{\lambda} M_{1}\left(G_{u, a}\right)}{n\left(G_{u, a}\right)}
$$

is then determined by

$$
\begin{aligned}
& g(a)=a^{\lambda+2}+a^{\lambda+1}\left(c_{1}+n\right)+a^{\lambda} n c_{1}+a c_{2}+n c_{2}- \\
& a^{2 \lambda+1}-a^{2}-a^{2 \lambda m}-a\left(c_{0}+m\right)-m c_{0} .
\end{aligned}
$$

For $0<\lambda<1$ we obtain that

$$
\lim _{a \rightarrow+\infty} \frac{g(a)}{a^{a+2}}=1,
$$

so that

$$
\lim _{a \rightarrow+\infty} g(a)=-\infty .
$$

Therefore, there exists a real number $A_{u}$ such that

$$
\frac{{ }^{\lambda} M_{1}\left(G_{u, a}\right)}{n\left(G_{u, a}\right)}>\frac{{ }^{\lambda} M_{2}\left(G_{u, a}\right)}{m\left(G_{u, a}\right)}
$$

for any $a>A_{u}$.

On the other hand, for $\lambda<0$ we have that

$$
\lim _{a \rightarrow+\infty} \frac{g(a)}{a^{2}}=-1,
$$

while for $1<\lambda$ we have that

$$
\lim _{a \rightarrow+\infty} \frac{g(a)}{a^{2+1}}=-1 .
$$

In both cases,

$$
\lim _{a \rightarrow+\infty} g(a)=-\infty .
$$

Therefore, there exists a real number $A_{u}$ such that

$$
\frac{{ }^{\lambda} M_{1}\left(G_{u, a}\right)}{n\left(G_{u, a}\right)}<\frac{{ }^{\lambda} M_{2}\left(G_{u, a}\right)}{m\left(G_{u, a}\right)}
$$

for any $a>A_{u}$.

Notice that the inequalities in Theorem 3 hold for all graphs, while in case $\lambda=1$ they hold in one direction for the counterexamples to Hansen's conjecture, and in another direction for other graphs. Since there are infinitely many graphs in both groups, one could consider $\lambda=1$ to be a sort of phase change for this inequality.

Acknowledgements. This work was supported by the research project TR036035 of the Serbian Ministry of Science and Technological Development. I would like to thank Prof. Dragan Stevanović for suggesting the research problem and helpful discussion while writing the paper. I am also grateful to the anonymous referees for their remarks, which led to the improved presentation of the results.

\section{REFERENCES}

1. I. Gutman and N. Trinajstić, Chem. Phys. Lett. 17 (1972) 535538.

2. I. Gutman, B. Ruščić, N. Trinajstić, and C. F. Wilcox, Jr., J. Chem. Phys. 62 (1975) 3399-3405.

3. S. Nikolić, G. Kovačević, A. Miličević, and N. Trinajstić, Croat. Chem. Acta 76 (2003) 113-124.

4. I. Gutman and K. Ch. Das, MATCH Commun. Math. Comput. Chem. 50 (2004) 83-92.

5. G. Caporossi and P. Hansen, Discrete Math. 212 (2000) 29-44.

6. P. Hansen and D. Vukičević, Croat. Chem. Acta 80 (2007) 165 168.

7. D. Vukičević and A. Graovac, MATCH Commun. Math. Comput. Chem. 57 (2007) 587-590.

8. B. Liu, Recent results in the Theory of Randic index, University of Kragujevac, Kragujevac, 2008, pp. 205-209.

9. A. Ilić and D. Stevanović, MATCH Commun. Math. Comput. Chem. 62 (2009) 681-687.

10. L. Sun and T. Chen, Discrete Appl. Math. 157 (2009) 16501654.

11. A. Miličević and S. Nikolić, Croat. Chem. Acta 77 (2004) 97-101.

12. D. Vukičević, MATCH Commun. Math. Comput. Chem. 57 (2007) 633-641.

13. D. Vukičević and A. Graovac, MATCH Commun. Math. Comput. Chem. 60 (2008) 37-44. 